\title{
The Effect of Surface Modifications on Chemical Composition of the Oil Palm and Coir Fibres
}

\author{
J.M. Jabar \\ Polymer and Textile Research Group, Chemistry Department, the Federal University of \\ Technology, Akure, Ondo State, Nigeria
}

\begin{abstract}
Chemical modifications of oil palm fibres (empty fruit bunch and palm kernel fibres) and coir fibres were studied using mercerization, alkaline bleaching and acetylation methods of fibre surface modification. The effect of these surface modifications on the weight and chemical composition of these fibres was investigated. Acetylation process led to $1.34-3.46 \%$ weight percentage gain (WPG) in the chemically modified fibres, while mercerization and alkaline bleached modified fibres are having $3.52-4.21 \%$ and $4.30-9.52 \%$ weight percentage loss (WPL) respectively. Comparative evaluation of the chemical compositions of chemically modified and unmodified fibres showed increase in cellulose from 3.29-8.31\%, $6.60-14.97 \%$ and $3.36-$ $5.76 \%$ in mercerized, alkaline bleached and acetylated fibres respectively. Hemicellulose content increased from $11.47-14.89 \%$ only in acetylation process, while lignin and pectin content decreased in all modification methods. These chemical modifications were confirmed by FT-IR spectroscopic and SEM methods of chemical analysis carried out on both chemically modified and unmodified fibres.
\end{abstract}

Keywords: Acetylation; Chemical; Fibre; Spectroscopic; Mercerization; Modification

\section{INTRODUCTION}

Fibre is any substance that has it length to be at least hundred times size of its breadth. It can be artificial or natural (Hassan et al. 2010). Artificial fibres are the man-made fibres, such as e glass, mica, carbon, aramid fibres etc, while the natural ones are those fibres from nature (Mehdi et al. 2009). In general, natural fibres can also be subdivided as to their origin: plants, animals, or minerals (Bhat et al. 2011). The sub-division in plant fibres are bast, stalk, leaf, seed and fruit fibres (Suddell, 2008). Oil palm and coconut trees contain many fibres, but coir fibre (CCF) from coconut, empty fruit bunch (EFB) and palm kernel (PKF) from oil palm belong to the class of fruit fibres. The major chemical compositions in these fibres are pectin, hemicelluloses, lignin and cellulose (Bledzki et al. 2008). Natural fibre's compositions may vary with nature of the plant, geographical location, growing condition and chemical determination methods. This variation may be noticed even in the same kind of fibre (Rakesh et al. 2011).

Pectin is a collective name for heteropolymers in natural fibre (Maya and Rajesh, 2008). Hemicellulose comprises of a group of polysaccharides that composed of a combination of 5- and 6carbon ring sugars. It exhibits a considerable degree of chain branching containing pendant side groups, giving rise to its non crystalline nature and the degree of polymerization of 50-300 repeat units. Hemicellulose forms the supportive matrix for cellulose micro fibrils. It is very hydrophilic in nature due to the presence of many hydroxyl groups in the repeat units. The availability of acetyl group in the monomeric units also makes the hemicellulose partially soluble in water but soluble in alkali and easily hydrolyzed in acids (Frederick and Norman, 2004). Lignin is a complex hydrocarbon polymer with both aliphatic and aromatic constituents, totally insoluble in most of solvents and cannot be broken down into monomeric units. Lignin is a compound that gives rigidity to the plants, thought to be a complex, three-dimensional copolymer of aliphatic and aromatic constituents with very high molecular weight. Five hydroxyl, five methoxyl and carbonyl groups per building unit have been identified in lignin. Although, some research work show that lignin molecule are derivatives of 4hydroxy-3-methoxy phenylpropane and considered to be thermoplastic polymer having a glass transition temperature of around $90^{\circ} \mathrm{C}$ and melting temperature of around $170^{\circ} \mathrm{C}$ (Olesen and Plackett, 1999). I t is totally amorphous and hydrophobic in nature. It is not hydrolyzed by acids, but soluble in hot alkali, readily oxidized and easily condensable with phenol (Rowell et al. 1996). Cellulose is a 


\section{J.M. Jabar}

natural polymer consisting of $\mathrm{D}$-anhydroglucose $\left(\mathrm{C}_{6} \mathrm{H}_{11} \mathrm{O}_{5}\right)$ repeating units joined by $\beta-1,4$ glucopyranose linkage at $\mathrm{C}_{1}$ and $\mathrm{C}_{4}$ positions. Cellulose is resistant to strong alkali and oxidizing agents but is easily hydrolyzed by hot acid to water-soluble sugars. The degree of polymerization is between 500 and 30,000. Each repeating unit contains three hydroxyl groups and their ability to form hydrogen bond play a major role in directing the crystalline packing (the crystal nature). Cellulose, when use to reinforced hydrophobic matrices; the result is a very poor interfacial adhesion and poor resistant to moisture absorption, because of it hydrophilic nature (Maya and Rajesh, 2008). These natural fibres' limitation can be minimized through surface treatment, by using either chemical compatibilisers/coupling agents, grafting of polymer matrix or chemical modification agents on the lignocellulosic fibres (Khalil et al. 2001). These surface treatments have significant effect on chemical compositions of the natural fibre, which in-turn leads to reduction in hydrophilic nature of the fibre and improvement in dimensional stability, interfacial adhesion and environmental degradation of the natural fibre (Bledzki et al. 2008). In the present research work, the effect of the mercerization, alkaline bleaching and acetylation on empty fruit bunch (EFB), palm kernel (PKF) and coir fibres' (CCF) chemical compositions was investigated.

\section{EXPERIMENTAL PROCEDURES}

\subsection{Materials}

The coir fibres used were obtained from cassava avenue, the Federal University of Technology, Akure (FUTA) road Akure. The empty fruit bunch and palm kernel fibres were obtained from FUTA research farm, Akure, Ondo state, Nigeria. Reagents used were of AnalaR grade obtained from Rovet Chemical store, Benin and Chemistry Department, FUTA.

\subsection{Surface Modification}

Coir (CCF), empty fruit bunch (EFB) and palm kernel fabres (PKF) (about $0.500 \mathrm{~g}$ each) were pretreated with $2 \%$ detergent solution in liquor ratio $1: 200$ at $80^{\circ} \mathrm{C}$ for $1 \mathrm{~h}$, washed with distilled water and finally oven dry at $105^{\circ} \mathrm{C}$ to a constant weight. The pretreated fibres were then divided into four portions; the first portion was not further treated and labeled unmodified (UCCF, UEFB and UPKF).

The second portion of the CCF, EFB and PKF fibres (about 0.050-0.100 g each) was soaked with continual stirring in $\mathrm{NaOH}(5 \%)$ solution in liquor ratio 1:200 for $2 \mathrm{~h}$ before washing with distilled water to be free of alkaline, oven dry to a constant mass and labeled mercerized (MCCF, MEFB and MPKF).

The third portion of the CCF, EFB and PKF fibres (about $0.050-0.100 \mathrm{~g}$ each) was soaked in bleaching solution containing $\mathrm{H}_{2} \mathrm{O}_{2}(2 \%)$ in $\mathrm{NaOH}(25 \%)$ with continual stirring in liquor ratio 1:200 for $2 \mathrm{~h}$, washed with distilled water to be bleaching solution free, oven dried to a constant mass at $105^{\circ} \mathrm{C}$ and tagged bleached fibres (BCCF, BEFB and BPKF).

The fourth portion of the CCF, EFB and PKF fibres (about 0.050-0.100 g each) was soaked in acetic acid solution (10\%) containing a drop of conc. $\mathrm{H}_{2} \mathrm{SO}_{4}$ as catalyst, stirred for $1 \mathrm{~h}$ at $60^{\circ} \mathrm{C}$ in liquor ratio 1:200, decanted off at the end of reaction time. This was followed by introduction of acetic anhydride solution $(10 \%)$ containing a drop of conc. $\mathrm{H}_{2} \mathrm{SO}_{4}$ as catalyst in liquor ratio $1: 200$ at $60^{\circ} \mathrm{C}$, stirred continually for $1 \mathrm{~h}$, washed with distilled water to be acid free, oven dried to a constant weight and tagged acetylated fibres (ACCF, AEFB and APKF).

\subsection{Determination of Chemical Composition of Modified and Unmodified Coconut Coir, Empty Fruit Bunch and Palm Kernel Fibres}

Percentage pectin, hemicellulose, lignin, holocellulose, and cellulose in chemically modified and unmodified CCF, EFB and PKF were determined to show the effect of the above described chemical modifications on the chemical composition of the fibres.

\subsubsection{Pectin Content}

The oven dried fibre samples (about $0.050-0.100 \mathrm{~g}$ each) were weighed in an extraction thimble and placed in Soxhlet extraction unit. A mixture of ethanol and benzene was used as extracting solvent at ratio 1:4, liquor ratio 1:200 and extraction process was done for a period of $3 \mathrm{~h}$. After extraction, the sample was rinsed with ethanol and hot water, oven dried for $2 \mathrm{~h}$ at the temperature of $105^{\circ} \mathrm{C}$ and finally dried up to constant weight at $15 \mathrm{~min}$ interval. The percentage pectin was calculated from the weight of oven dried test sample and soxhlet extracted sample. This method was used for both chemically modified and unmodified fibres in each of the samples (Khalil et al. 2001). 


\subsubsection{Lignin Content}

The oven dried fibre samples (about $0.050 \mathrm{~g}$ each) were weighed in a conical flask and $72 \%$ sulphuric acid was added at liquor ratio 1:200. The mixture was stirred continually for $2 \mathrm{~h}$ at room temperature and $200 \mathrm{~mL}$ of distilled water was added to the mixture at the end of $2 \mathrm{~h}$ reaction time. The mixture was boiled for $2 \mathrm{~h}$, cooled and left open for $24 \mathrm{~h}$. The mixture was then filtered; residue transferred to the crucible and washed with hot water repeatedly until it was acid free. The collected residue was dried at $105^{\circ} \mathrm{C}$ in an oven for $2 \mathrm{~h}$ and finally dried up to constant weight at $15 \mathrm{~min}$ interval. The weight of the residue obtained was taken as mass of lignin in the fibre sample and this was used to calculated percentage lignin in both chemically modified and unmodified fibre samples (Bledzki et al. 2008).

\subsubsection{Hemicellulose Content}

The oven dried fibre samples (about $0.050-0.100 \mathrm{~g}$ each) were placed in a conical flask and sodium hydroxide solution (5\%) was added in liquor ratio 1:200 at room temperature for $2 \mathrm{~h}$ of continual stirring. At the end of reaction time, the sample was rinsed with distilled water, dried at $105^{\circ} \mathrm{C}$ in an oven for $2 \mathrm{~h}$ and finally dried up to constant weight at $15 \mathrm{~min}$ interval. The hemicellulose was calculated by subtracting the weight of oven dried test sample from the initial weight of the fibre sample (Oladele et al. 2000).

\subsubsection{Cellulose Content}

The content of cellulose was determined by soaking about $0.050 \mathrm{~g}$ of each of the fibres in $\mathrm{NaOH}$ solution (25\%) in liquor ratio 1:200 at room temperature with continual stirring for $2 \mathrm{~h}$. At the end of treatment time, $\mathrm{NaOH}$ solution was decanted, fibre washed with distilled water to be alkali free, oven dry for $2 \mathrm{~h}$ at $105^{\circ} \mathrm{C}$ and dry to a constant weight at $15 \mathrm{~min}$ interval. The weight of the residue was used to calculate percentage cellulose in the fibre sample (Rakeh et al. 2011).

\subsection{FT-IR Spectroscopy}

The technique used to confirm the product of the chemical modification of raw fibres by the mercerization, alkali bleaching and esterification is Fourier Transform Infrared Spectroscopy (FTIR). The infrared spectra of the fibers (modified and unmodified) were obtained by using ground fibers mixed with potassium bromide at ratio 1:100 in a mortar pestle. The mixture was then taken in a dice of specific dimension to form pellet by pressing with a hand press machine, placed on the sample holder and the IR spectrometer operated at spectral range $4000-350 \mathrm{~cm}^{-1}$. The IR spectrum obtained in this study is presented in the results and discussion section.

\subsection{Surface Morphology of the Fibres}

The scanning electron micrographs of the samples were taken in a XL 20 Philips SEM. The surface morphology of the fibres was studied with the microscope operated at $10.0 \mathrm{kV}$. The samples were coated with a $10 \mathrm{~nm}$ thick layer of gold.

\section{RESULTS AND DISCUSSION}

\subsection{Chemical Compositions of Unmodified Fibres}

The natural fibre major chemical compositions are cellulose, lignin, hemicellulose and pectin. Table 1 shows the percentage composition of unmodified empty fruit bunch (UEFB) and unmodified palm kernel fibres (UPKF) in order cellulose > lignin > hemicellulose > pectin, while unmodified coir fibres (UCCF) displayed order lignin > cellulose > hemicellulose > pectin. These observations agreed with Rakesh et al., (2011), in chemical modifications of natural fibre for composite material and Rozman et al., (2003), in flexural and impact properties of oil palm empty fruit bunch (EFB) polypropylene composites - the effect of maleic anhydride chemical modification of EFB.

Table1. Chemical Composition of Unmodified Coconut Coir (UCCF), Oil Palm Empty Fruit Bunch (UEFB) and Palm Kernel Fibres (UPKF)

\begin{tabular}{|l|l|l|l|}
\hline Composition & UCCF & UEFB & UPKF \\
\hline Pectin $(\mathrm{g})$ & $1.93 \pm 0.05$ & $2.00 \pm 0.06$ & $2.44 \pm 0.41$ \\
\hline Hemicellulose $(\mathrm{g})$ & $9.01 \pm 0.07$ & $13.71 \pm 0.12$ & $14.05 \pm 0.39$ \\
\hline Lignin $(\mathrm{g})$ & $47.69 \pm 0.63$ & $21.64 \pm 0.33$ & $30.00 \pm 0.74$ \\
\hline Cellulose $(\mathrm{g})$ & $40.77 \pm 0.63$ & $61.63 \pm 0.17$ & $51.82 \pm 1.49$ \\
\hline Total & 99.40 & 98.98 & 98.31 \\
\hline
\end{tabular}




\subsection{Chemical Compositions of Mercerized Fibres}

The modifications that occurred in mercerization of CCF, EFB and PKF fibres were the removal and replacement of hydrogen bonding with sodium bonding in the network structure of hemicellulose through hydrolysis in alkaline solution to form alkali-cellulose. The alkali-cellulose was washed with distilled water to give alkaline free fibres (Scheme 1). The mercerized fibres are more hydrophobic with wider pore sizes than raw fibres. Therefore, the resulting fibres might be more compatible with higher reinforcement power in matrix composites according to Maya and Rajesh, (2008).

Fibre- $-\mathrm{OH}+\mathrm{NaOH} \rightarrow$ Fibre $--\mathrm{O}-\mathrm{Na}++\mathrm{H}_{2} \mathrm{O} \rightarrow$ Fibre $--\mathrm{O}-\mathrm{H}+\mathrm{NaOH}$

Scheme1. Mercerization of CCF, EFB and PKF fibres (Maya and Rajesh, 2008)

Mercerization of CCF, EFB and PKF fibres led to fibre weight percentage loss (WPL) as a result of hydrolysis of hemicellulose (Table 2). This WPL observed is a proof of mercerization of the fibres and it was further confirmed by FT-IR spectra in Fig. 1(B). This observation agreed with Bhat et al. (2011), in morphological, spectroscopic and thermal properties of alkali-treated and chemical modified oil palm empty fruit bunch fibre and oil palm frond fibres. Although literature showed that mercerization of natural fibres has great effect on hemicellulose, but it's been observed in this work that it also has effect on pectin, lignin and cellulose (Table 2). Pectin, hemicellulose and lignin are the cementing materials in the natural fibres that got dissolved as a result of alkaline treatment (Laly et al. 2002).

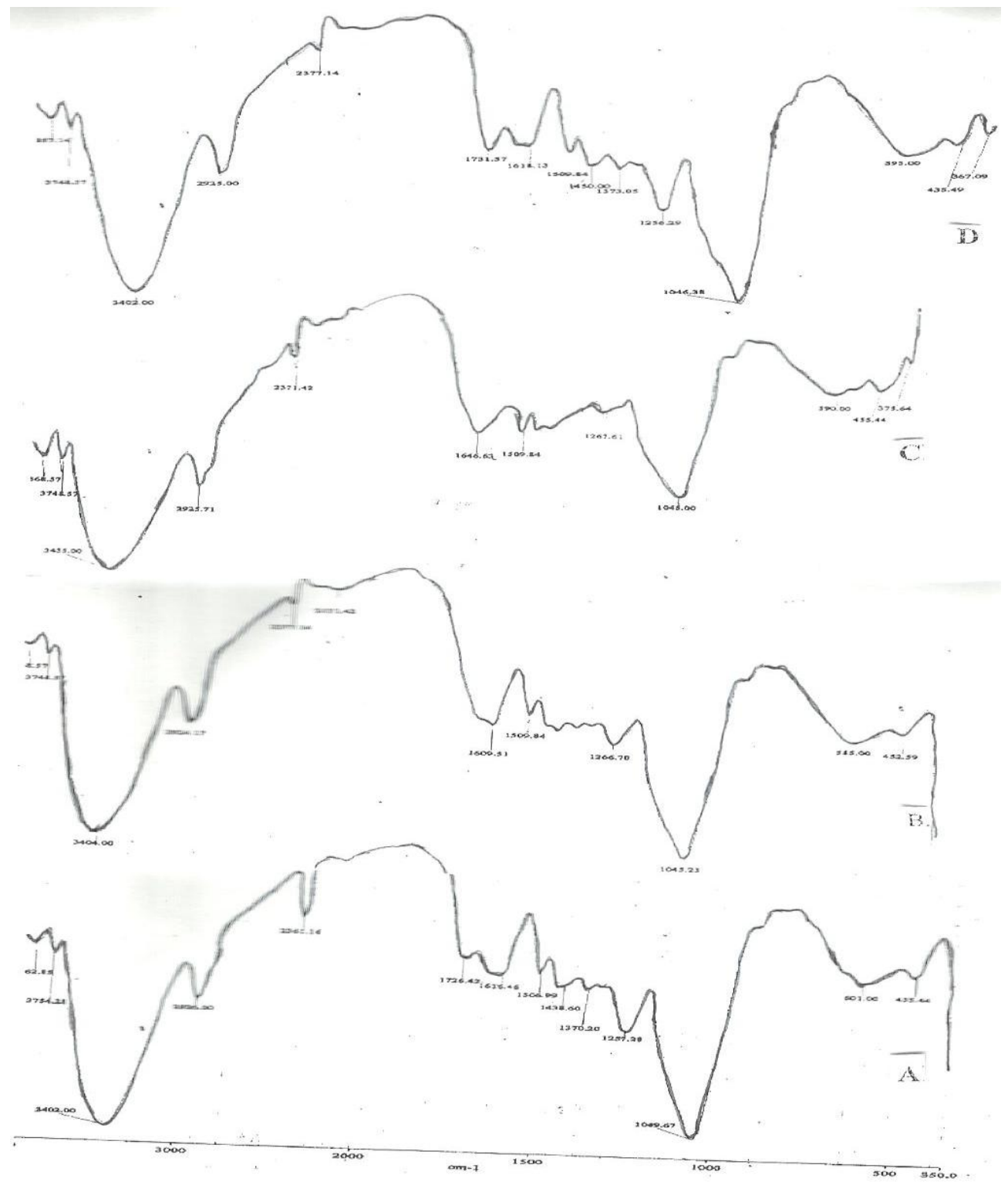

Figure1. IR spectra of unmodified $(A)$, mercerized $(B)$, alkaline bleached $(C)$ and acetylated $(D)$ empty fruit bunch fibres 
The Effect of Surface Modifications on Chemical Composition of the Oil Palm and Coir Fibres

Table2. Effect of mercerization on chemical composition of the CCF, EFB and PKF fibres

\begin{tabular}{|l|l|l|l|l|}
\hline Fibre/Composition & $\mathrm{W}_{1}(\mathrm{~g})$ & $\mathrm{W}_{2}(\mathrm{~g})$ & $\mathrm{WPG}(\%)$ & $\mathrm{WPL}(\%)$ \\
\hline CCF & $\mathbf{0 . 0 5 2 2}$ & $\mathbf{0 . 0 5 0 0} \pm \mathbf{0 . 0 2}$ & - & $\mathbf{4 . 2 1}$ \\
\hline Pectin & 0.0475 & $0.0474 \pm 0.01$ & - & 10.91 \\
\hline Hemicellulose & 0.0475 & $0.0471 \pm 0.02$ & - & 9.35 \\
\hline Lignin & 0.0237 & $0.0230 \pm 0.02$ & - & 6.19 \\
\hline Cellulose & 0.0236 & $0.0244 \pm 0.03$ & 8.31 & - \\
\hline EFB & $\mathbf{0 . 0 4 8 3}$ & $\mathbf{0 . 0 4 6 6} \pm \mathbf{0 . 0 5}$ & - & $\mathbf{3 . 5 2}$ \\
\hline Pectin & 0.0417 & $0.0416 \pm 0.02$ & - & 11.99 \\
\hline Hemicellulose & 0.0208 & $0.0205 \pm 0.04$ & - & 10.52 \\
\hline Lignin & 0.0199 & $0.0198 \pm 0.02$ & - & 2.321 \\
\hline Cellulose & 0.0197 & $0.0201 \pm 0.02$ & 3.29 & - \\
\hline PKF & $\mathbf{0 . 1 0 2 0}$ & $\mathbf{0 . 0 9 8 3} \pm \mathbf{0 . 0 2}$ & - & $\mathbf{3 . 6 3}$ \\
\hline Pectin & 0.0877 & $0.0874 \pm 0.08$ & - & 14.02 \\
\hline Hemicellulose & 0.0877 & $0.0864 \pm 0.06$ & - & 10.55 \\
\hline Lignin & 0.0438 & $0.0434 \pm 0.02$ & - & 3.04 \\
\hline Cellulose & 0.0438 & $0.0449 \pm 0.07$ & 4.85 & - \\
\hline
\end{tabular}

Where: W1 = Weight of fibres before modification; W2 = Weight of fibres after modification; WPG = Weight percentage gain; $W P L=$ Weight percentage loss

\subsection{Chemical Compositions of Alkali Bleached Fibres}

The bleaching action of hydrogen peroxide under alkaline condition is attributed to the hydroperoxide anion $\left(\mathrm{HOO}^{-}\right)$, hydroxyl radicals $\left(\mathrm{HO}^{*}\right)$ or oxygen radical $\left(\mathrm{O}^{*}\right)$ which is responsible for the elimination of hemicellulose and delignication of lignocelluloses materials (Ray et al. 2001; Sun et al. 2002). Scheme 2 shows chemical representation of fibres' alkali bleaching.

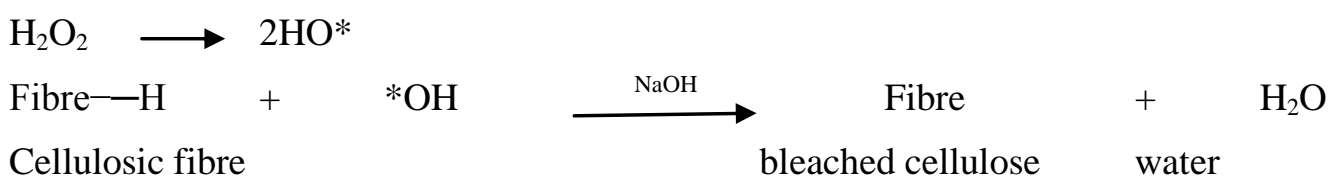

Scheme2. Alkali bleaching of natural fibre (Rakesh et al., 2008)

The modifications that occurred in alkali bleaching of CCF, EFB and PKF fibres were the removal of chromophoric groups from lignin, carbonyl groups from pectin and hemicellulose. This observation agreed with the findings of Georgopoulos et al. (2005); Habibi et al. (2008). The elimination of these chemical compounds led to fibre weight percentage loss (WPL) (Table 3). This observation is supported by FT-IR spectra in Fig. 1(C).

Table3. Effect of alkaline bleaching on chemical composition of the CCF, EFB and PKF fibres

\begin{tabular}{|l|l|l|l|l|}
\hline Fibre/Composition & $\mathrm{W}_{1}(\mathrm{~g})$ & $\mathrm{W}_{2}(\mathrm{~g})$ & $\mathrm{WPG}(\%)$ & $\mathrm{WPL}(\%)$ \\
\hline CCF & $\mathbf{0 . 0 8 9 3}$ & $\mathbf{0 . 0 8 0 8} \pm \mathbf{0 . 0 4}$ & - & $\mathbf{9 . 5 2}$ \\
\hline Pectin & 0.0426 & $0.0425 \pm 0.02$ & - & 12.16 \\
\hline Hemicellulose & 0.0467 & $0.0459 \pm 0.03$ & - & 19.01 \\
\hline Lignin & 0.0236 & $0.0219 \pm 0.02$ & - & 15.10 \\
\hline Cellulose & 0.0213 & $0.0226 \pm 0.02$ & 14.97 & - \\
\hline EFB & $\mathbf{0 . 0 6 2 8}$ & $\mathbf{0 . 0 6 0 1} \pm \mathbf{0 . 0 3}$ & - & $\mathbf{4 . 3 0}$ \\
\hline Pectin & 0.0491 & $0.0490 \pm 0.01$ & - & 10.18 \\
\hline Hemicellulose & 0.0491 & $0.0481 \pm 0.02$ & - & 14.86 \\
\hline Lignin & 0.0245 & $0.0240 \pm 0.02$ & - & 9.43 \\
\hline Cellulose & 0.0246 & 0.0246 & 6.60 & - \\
\hline PKF & $\mathbf{0 . 1 0 0 1}$ & $\mathbf{0 . 0 9 3 2} \pm \mathbf{0 . 0 4}$ & - & $\mathbf{6 . 8 9}$ \\
\hline Pectin & 0.0691 & $0.0689 \pm 0.02$ & - & 11.86 \\
\hline Hemicellulose & 0.0691 & $0.0675 \pm 0.02$ & - & 16.48 \\
\hline Lignin & 0.0345 & $0.0335 \pm 0.03$ & - & 9.66 \\
\hline Cellulose & 0.0346 & $0.0366 \pm 0.02$ & $11.15 \pm 0.02$ & - \\
\hline
\end{tabular}

\subsection{Chemical Composition of Acetylated Fibres}

Acetylation is a kind of esterification reaction of natural fibres with weight percentage gain of the treated fibre as proof of acetylation (Bledzki et al. 2008; Khalil et al. 2001), confirmed by FT-IR 
spectra in Fig. 1(D). Acetylation reaction scheme is shown in Scheme 3, weight percentage gain and effect of acetylation on CCF, EFB and PKF fibres are shown in Table 4.

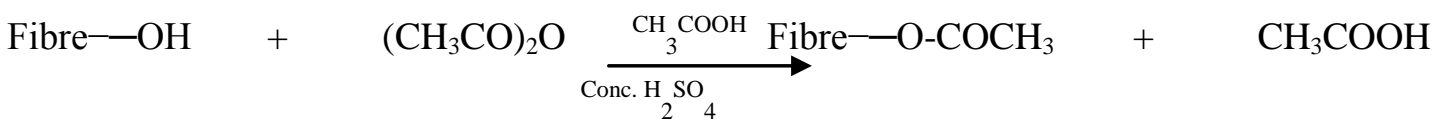

Cellulose Acetic anhydride Acetylated cellulose Acetic acid

Scheme3. Esterification of natural fibre (Bledzki et al., 2008; Khalil et al., 2001)

Acetylation of CCF, EFB and PKF fibres led into degradation of pectin and lignin in acetylating solution according to Medhi et al. (2009). This in turn resulted into reduction in weight of pectin and lignin as shown in Table 4. Formation of acetylated hemicellulose and cellulose in acetylation of $\mathrm{CCF}, \mathrm{EFB}$ and PKF fibres resulted into increase in weight of hemicellulose and cellulose content of the fibres (Table 4).

Table4. Effect of acetylation on Chemical composition of the CCF, EFB and PKF CCF, EFB and PKF fibres

\begin{tabular}{|l|l|l|l|l|}
\hline Fibre/Composition & $\mathrm{W}_{1}(\mathrm{~g})$ & $\mathrm{W}_{2}(\mathrm{~g})$ & $\mathrm{WPG}(\%)$ & $\mathrm{WPL}(\%)$ \\
\hline CCF & $\mathbf{0 . 0 4 9 1}$ & $\mathbf{0 . 0 5 0 8} \pm \mathbf{0 . 0 2}$ & $\mathbf{3 . 4 6}$ & - \\
\hline Pectin & 0.0489 & $0.0488 \pm 0.02$ & - & 10.60 \\
\hline Hemicellulose & 0.0484 & $0.0489 \pm 0.03$ & 11.47 & - \\
\hline Lignin & 0.0249 & $0.0245 \pm 0.02$ & - & 3.37 \\
\hline Cellulose & 0.0213 & $0.0218 \pm 0.01$ & 5.76 & - \\
\hline EFB & $\mathbf{0 . 0 4 6 5}$ & $\mathbf{0 . 0 4 7 6} \pm \mathbf{0 . 0 2}$ & $\mathbf{2 . 3 7}$ & - \\
\hline Pectin & 0.0488 & $0.0487 \pm 0.03$ & - & 10.25 \\
\hline Hemicellulose & 0.0462 & $0.0470 \pm 0.02$ & 12.63 & - \\
\hline Lignin & 0.0190 & $0.0186 \pm 0.03$ & - & 9.73 \\
\hline Cellulose & 0.0193 & $0.0197 \pm 0.02$ & 3.36 & - \\
\hline PKF & $\mathbf{0 . 0 9 7 2}$ & $\mathbf{0 . 0 9 8 5} \pm \mathbf{0 . 0 2}$ & $\mathbf{1 . 3 4}$ & - \\
\hline Pectin & 0.0976 & $0.0974 \pm 0.03$ & - & 8.40 \\
\hline Hemicellulose & 0.0956 & $0.0976 \pm 0.01$ & 14.89 & - \\
\hline Lignin & 0.0400 & $0.0391 \pm 0.02$ & - & 7.50 \\
\hline Cellulose & 0.0407 & $0.0418 \pm 0.01$ & 5.22 & - \\
\hline
\end{tabular}

\subsection{FT-IR Spectra}

The FT-IR spectra of the unmodified and chemically modified CCF, EFB and PKF fibres are similar. Therefore, spectra of EFB fibres show in Fig. 1 represent FT-IR spectra of unmodified and chemically modified lignocellulosic fibres. The absorbance peak in the $3400-3450 \mathrm{~cm}^{-1}$ attributed to stretching of O-H group in cellulose, lignin and hemicellulose in CCF, EFB and PKF fibres. The absorbance peaks 2920 to $2930 \mathrm{~cm}^{-1}, 1730-1750 \mathrm{~cm}^{-1}, 1410-1460 \mathrm{~cm}_{-1}$ and $1040-1060 \mathrm{~cm}^{-1}$ were due to stretching $\mathrm{C}-\mathrm{H}$ in aromatic ring and alkanes (Khalil et al. 2001), $\mathrm{C}=\mathrm{O}$ stretching of acetyl group in hemicellulose, lignin and the ester linkage of carboxylic group in lignin and/or hemicellulose (Bledzki et al. 2008), $\mathrm{CH}_{2}$ from cellulose and alcoholic $\mathrm{C}-\mathrm{O}$ stretch respectively can be seen clearly in unmodified fibres (Fig. 1A).

The major modifications done on CCF, EFB and PKF fibres by mercerization and alkali bleaching was lignin and/or hemicellulose removal in fibers, which led to the disappearance of an absorbance peak in the regions of 1740 to $1720 \mathrm{~cm}^{-1}$ (Fig. 1A-B) This observation confirmed degradation of carbonyl group in pectin, hemicellulose and aliphatic lignin according to Medhi et al. (2009), in chemical composition, crystallinity and thermal degradation of bleached and unbleached kenaf bast pulp and nanofibres.

In acetylation treatment, there were increase in the $\mathrm{C}=\mathrm{O}$ absorbance peaks as a result of replacement of $\mathrm{OH}$ with acetyl functional group in hemicellulose and amorphous cellulose to form acetylated hemicellulose and cellulose. This observation agreed with Khalil et al. (2001), in the effect of acetylation on interfacial shear strength between plant fibres and various matrices and Bledzki et al. (2008), in composites reinforced with cellulose based fibres.

\subsection{Morphological Study}

The micrographs of the fibres are shown in Plates 1-3. The unmodified fibres have rough surfaces and small pore sizes (Plates 1A, 2E and 3I). The surface roughness and small pore sizes of the unmodified 
fibres were due to the presence of impurities (Suradi et al. 2009). The chemical modification of the fibres might improve physical and mechanical interlocking of the natural fibres to the matrices leading to better interfacial bonding between fibres and matrices, as a result of increase in pore sizes according to Myrtha et al. (2008).

The mercerized fibres have most of their impurities and residual hemicellulose removed; appear cleaner with wider pore sizes and clearly seen silica nodules (Plates $1 \mathrm{~B}, 2 \mathrm{~F}$ and $3 \mathrm{~J}$ ) as a result of alkaline reaction with cementing materials of the fibres and splitting the fibers into finer filaments (Bhat et al. 2011).

The alkaline bleached CCF, EFB and PKF fibres have not only their lignin component degraded but also most of their hemicellulose removed with formation of widest pore sizes on the surfaces of the fibres (Plates 1C, 2G and 3K). These observations are in agreement with the findings made by Suradi et al. (2009) in influence of pre-treatment on the properties of lignocellulose based biocomposite.

After the modification with acetic anhydride, the surfaces of the fibres appear smoother with a little increase in surface pore sizes (Plates 1D, 2H and 3L) as a result of conversion of hydroxyl group on the fibres surface to hydrophobic acetyl group. This observation is in line with the one made by Khalil et al. (2001) in the effect of acetylation on interfacial shear strength between plant fibres and various matrices.
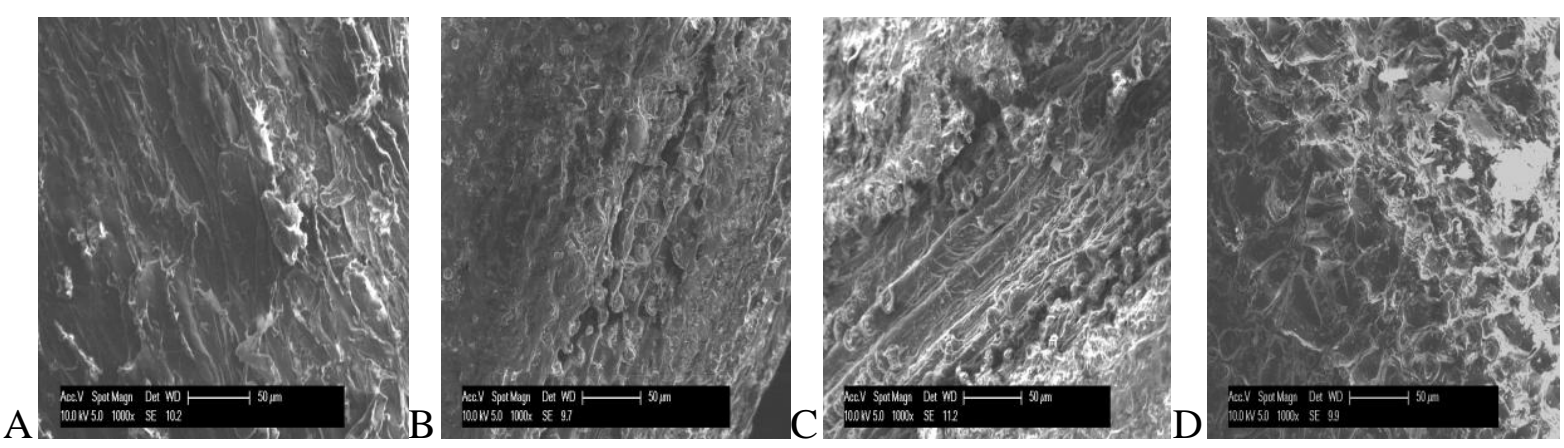

Plate1. $A, B, C$ and $D$ are SEM images of $U C C F, M C C F, B C C F$ and ACCF fibres respectively
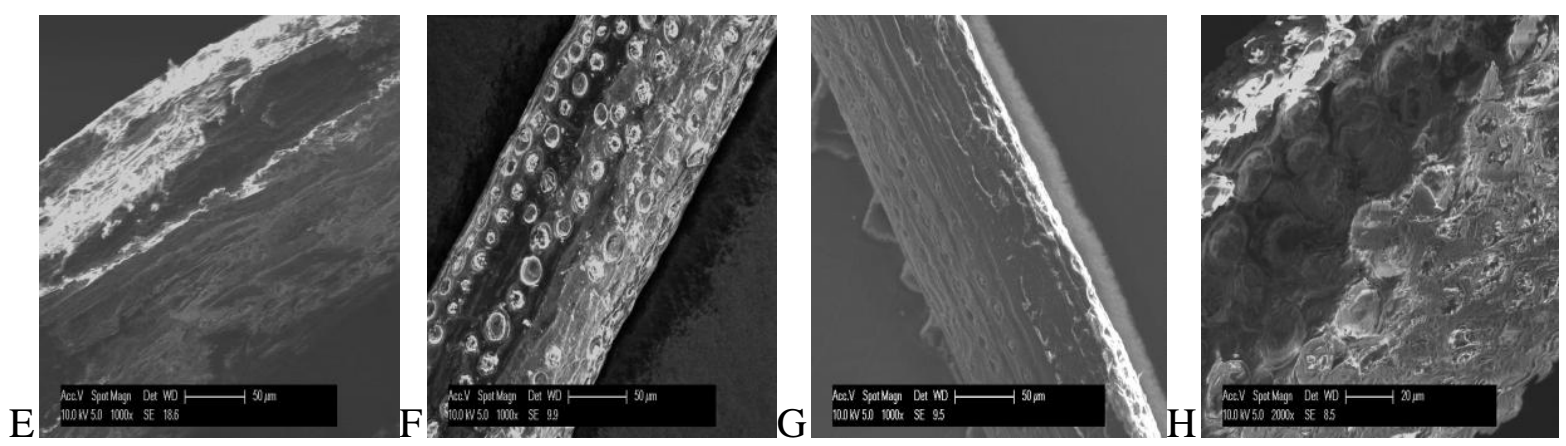

Plate2. $E, F, G$ and $H$ are SEM images of $U E F B, M E F B, B E F B$ and $A E F B$ fibres respectively
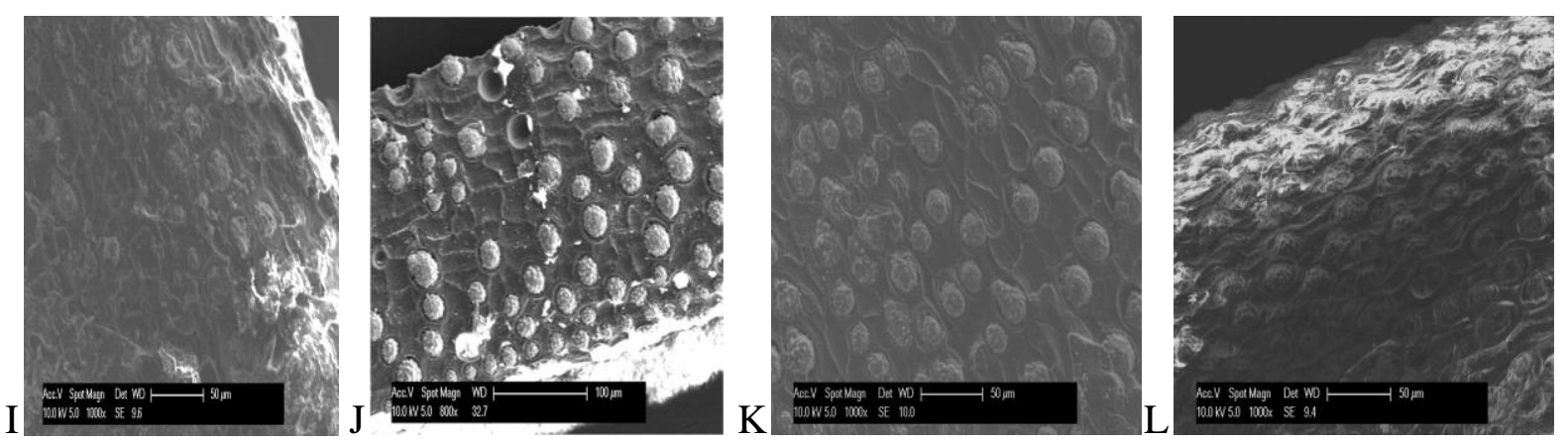

Plate3. $I, J, K$ and L are SEM images of $U P K F, M P K F, B P K F$ and APKF fibres respectively

Where: $U C C F=$ Unmodified coconut coir fibres, $M C C F=$ Mercerized coconut coir fibres, BCCF= Alkali bleached coconut coir fibres, 
ACCF $=$ Acetylated coconut coir fibres

$U E F B=$ Unmodified empty fruit bunch fibres, MEFB = Mercerized empty fruit bunch fibres, BEFB= Alkali bleached empty fruit bunch fibres, ACCF = Acetylated empty fruit bunch fibres

$U P K F=$ Unmodified palm kernel fibres, MPKF = Mercerized palm kernel fibres, BPKF = Alkali bleached palm kernel fibres,

APKF = Acetylated palm kernel fibres

\section{CONCLUSION}

The mercerization and alkali bleaching of CCF, EFB and PKF fibres led to decrease in weight of the fibres, while acetylation resulted into increase in weight of the fibres as proof of mercerization/alkali bleaching and acetylation respectively.

The percentage composition of all the chemically modified fibres as compare to unmodified fibres contain pectin in order untreated fibres (UT) $>$ acetylated fibres $(\mathrm{E})>$ mercerized fibres $(\mathrm{M})>$ alkali bleached fibres (B). Hemicellulose is in order $\mathrm{E}>\mathrm{UT}>\mathrm{M}>\mathrm{B}$. Cellulose is in order $\mathrm{B}>\mathrm{M}>\mathrm{E}>\mathrm{UT}$, while lignin is in order $\mathrm{UT}>\mathrm{M}>\mathrm{B}>\mathrm{E}$ for EFB and PKF. But CCF fibres has lignin content in order $\mathrm{UT}>\mathrm{M}>\mathrm{E}>\mathrm{B}$.

The chemically modified natural fibres were of cleaner surfaces with higher porosity and wider pore sizes than unmodified fibres.

\section{REFERENCES}

Bhat I, Abdul Khalil HPS, Ismail H, Alshammari T (2011) Morphoogical, spectroscopic and thermal properties of alkali-treated and chemical modified oil palm empty fruit bunch fibre and oil palm frond fibres: a comparative study. Biores 6(4):467-4685

Bledzki AK, Mamun AA, Lucka-Gabor M, Gutowski VS (2008) The effect of acetylation on properties of flax fibre and its polypropylene composites. Expr Poly Letter 2(6):413-422

Frederick TW, Norman W (2004) Natural fibres plastics and composites.

Kluwer Academic, New York Georgopoulos ST, Tarantili PA, Avgerinos E, Andreopoulos AG, Koukios EG (2005) Thermoplastic polymers reinforced with fibrous agricultural residues. Poly Degra and Stab 90:303-312

Habibi Y, El-Zawawy WK, Ibrahim MM, Dufresne A (2008) Processing and characterization of lignins solubilized during alkaline peroxide treatment of barley. Composi 32:119-127

Hassan A, Salema AA, Ani FN, Bakar AA (2010) A review on oil palm empty fruit bunch fibrereinforced polymer composite materials. Polym Composites 31:2079-2101

Khalil HPSA, Ismail B, Rozman HD, Ahmad MN (2001) The effect of acetylation on interfacial shear strength between plant fibres and various matrices. Eur Poly J 37:1037-1045

Laly A, Pothan JG, Sabu T (2002) Effect of fiber surface treatments on the fiber-matrix interaction in banana fiber reinforced polyester composites. Composi Int 9(4): 335-353

Maya JJ, Rajesh DA (2008) Recent developments in chemical modification and characterization of natural fiber 25reinforced composites. Poly Composi $187-207$

Medhi J, Jonoobi H, Alireza S, Manjusri MK (2009) Chemical composition, crystallinity and thermal degradation of bleached and unbleached kenaf bast pulp and nanofibres. Biores 4(2): 62-639

Myrtha K, Holia O, Abdullah DAH, Anung S, (2008) Effect of oil palm empty fruit bunch fibre on the physical and mechanical properties of fibre glass reinforced polyester resin. J Bio Sci 8(1):101-106

Oladele IO, Omotoyinbo JA, Adewara JOT (2010) Investigating the effect of chemical treatment on the constituents and tensile properties of sisal fibre. J Min Mat Charact Eng 9(6):569-582

Olesen PO, Plackett DV (1999) Perspectives on the performance of natural plant fibres. Natural Fibres Performance Forum, Copenhagen, May, 27-28

Rakesh K, Sangeeta O, Aparna S (2011) Chemical modification of natural fibre for composite material. Der Chem Snica 2(4):219-228

Ray D, Sarkar BK, Rana AK, Bose NR (2001) The mechanical properties of fibers from 1 Egyptian agro-industrial residues. Comp Sci Techn 68:1877-1885 
Rowell RM, Young RA, Rowell JK (1996) Paper and composites from agro-based resources. CRC, Boca Roton

Rozman HD, Saad MJ, Mohd IZA. 2003, Flexural and impact properties of oil palm empty fruit bunch (EFB)- polypropylene composites - the effect of straw. Eur Poly J 38:1399-1407

Suddell BC (2008) Keynote Presentation: Innovative natural composite materials, natural materials event, Institute of Materials, Minerals and Mining. 7th March, London

Sun RC, Sun XF, Fowler P, Tomkinson J (2002) Structural and physico-chemical characterization of lignins solubilized during alkaline peroxide treatment of barley. Compos 32: 119-127

Suradi SS, Yunus RM, Beg MDH, Yusof ZAM (2009) Influence of pre-treatment on the properties of lignocellulose based biocomposite. National Conference on Postgraduate Research held on 1st October at UMP Conference Hall, Universiti Malaysia Pahang, Malaysia 67-78 